\title{
$G$-linear sets and torsion points in definably compact groups
}

\author{
Margarita Otero* \\ Ya'acov Peterzil \\ Universidad Autónoma de Madrid University of Haifa
}

August 11, 2021

\begin{abstract}
Let $G$ be a definably compact group in an o-minimal expansion of a real closed field. We prove that if $\operatorname{dim}(G \backslash X)<\operatorname{dim} G$ for some definable $X \subseteq G$ then $X$ contains a torsion point of $G$. Along the way we develop a general theory for so-called $G$-linear sets, and investigate definable sets which contain abstract subgroups of $G$.
\end{abstract}

Keywords: o-minimality, definable group, torsion point. Mathematics Subject Classification 2000: 03C64

\section{Introduction}

We prove:

Theorem 1.1. Let $G$ be a definably compact group in an o-minimal expansion of a real closed field. If $X \subseteq G$ is a definable large set (i.e. $\operatorname{dim}(G \backslash X)<\operatorname{dim} G)$ then $X$ contains a torsion point of $G$.

This is a weak approximation to the conjecture that every generic set in a definably compact group contains a torsion point, a conjecture which itself follows from the "compact domination conjecture" from [4].

The proof starts with the abelian case, where we first develop, in ominimal expansions of an ordered group, a notion of a coset of a definable local subgroup (a $G$-linear set). We then use tools from Algebric Topology, in expansions of real closed fields, to conclude the theorem.

*Partially supported by GEOR MTM2005-02568 and Grupos UCM 910444 


\section{$2 \quad G$-linear sets}

In this section $\mathcal{M}$ will denote an o-minimal expansion of an ordered group. By definable we mean definable in $\mathcal{M}$. $G$ will denote a definable abelian group. All topological concepts are with respect to the group topology such $G$ is equipped with. (We believe that most, if not all, of the work below can be developed in an arbitrary definable group, but for simplicity we limit ourselves to the commutative setting.)

Definition 2.1. 1) Given two sets $X, Y \subseteq G$ and $a \in G$, we say that $X$ and $Y$ have the same germ at $a$, in notation $X={ }_{a} Y$, if there exists an open neighborhood $U$ of a such that $X \cap U=Y \cap U$. We say that the germ of $X$ at $a$ is contained in the germ of $Y$ at $a$, in notation $X \subseteq_{a} Y$, if there exists an open $U \ni$ a such that $X \cap U \subseteq Y \cap U$.

2) Given $X \subseteq G$ and $g, h \in G$, we say that the germ of $X$ at $g$ is $G$ equivalent to the germ of $X$ at $h$ if $X-g={ }_{0} X-h$. (Note that if $X$ is a definable set then we obtain in this way a definable equivalence relation on G.)

Definition 2.2. Let $X$ be a definable subset of $G$.

1) $X$ is called $G$-linear if for every $g, h \in X$ we have $X-g={ }_{0} X-h$. Given $g \in X$, we say that $X$ is locally $G$-linear at $g$ if there exists an open $U \ni g$ such that for every $h \in U \cap X$ we have $X-g={ }_{0} X-h$.

Two $G$-linear sets $X, Y \subseteq G$ are called $G$-equivalent if for every $g \in X$, $h \in Y$, we have $X-g={ }_{0} Y-h$.

2) Let $X$ be a $G$-linear set. A definable $Y \subseteq G$ is called a $G$-subset of $X$ if for every $h \in Y$ and $g \in X$, we have $Y-h \subseteq_{0} X-g$.

Example 2.3. Let $G$ be a definable abelian group and $H$ a definable subgroup. If $X$ is a relatively open subset of a coset of $H$ then $X$ is $G$-linear. If $Y$ is any definable subset of a coset of $H$ then $Y$ is a G-linear subset of $X$.

We observe without a proof (we will not be using it): If $\mathcal{M}$ expands a real closed field then $Y$ is a $G$-subset of the $G$-linear set $X$ if and only if at every smooth point $y \in Y$, the tangent space of $Y$ at $y$ is a subset of the tangent space of $X$ at any of its points point.

Lemma 2.4. If $X$ is definably connected and locally $G$-linear at every $g \in X$ then $X$ is $G$-linear.

Proof. Because $X$ is locally $G$-linear at every point the $G$-equivalence class of every $g \in X$ is relatively open in $X$. But then it is also relatively closed 
(since its complement is a union of open sets), so by definable connectedness there exists only one $G$-equivalence class, so $X$ is $G$-linear.

Definition 2.5. Given $X \subseteq G$, we let

$$
X_{\text {lin }}=\{g \in X: X \text { is locally } G \text {-linear at } g\} .
$$

Notice that $X_{\text {lin }}$ is a relatively open (possibly empty) subset of $X$. By the last lemma, every definably connected component of $X_{l i n}$ is a $G$-linear set, but $X_{\text {lin }}$ itself might not be $G$-linear (E.g., if $X=H_{1} \cup H_{2}$ is the union of two definable subgroups of $G$, none containing the other, then $X_{\text {lin }}=X \backslash\left(H_{1} \cap H_{2}\right)$ is not a $G$-linear set). It is easy to see that $X_{\text {lin }}$ is itself $G$-linear if and only if all its definably connected components are $G$-equivalent.

Notation We denote by $X_{\text {lin }}^{\max }$ the union of all components of $X_{\text {lin }}$ of maximal dimension.

\section{Infinitesimals}

It might be easier here to use the language of infinitesimals: We move to an $|M|^{+}$-saturated elementary extension $\mathcal{N}$ of $\mathcal{M}$. For $g \in G(\mathcal{M})$, we denote by $\nu_{g}$ the intersection of all $\mathcal{M}$-definable open neighborhoods of $g$ (with respect to the group topology) in the structure $\mathcal{N}$. For $X \subseteq G$, we write $\nu_{g}(X)=\nu_{g} \cap X$. Notice that for $X, Y \subseteq G$ and $g, h \in G, \nu_{g}(X)=\nu_{g}(Y)$ iff $X={ }_{g} Y$, and $\nu_{g}(X)-g=\nu_{h}(X)-h$ iff $X-g={ }_{0} X-h$.

We will be using the following simple observation: If $X, Y$ are $A$-definable sets, and $x$ is generic in $X$ and in $Y$ over $A$ then $\nu_{x}(X) \subseteq Y$.

Lemma 2.6. $X$ is locally $G$-linear at $g \in X$ if and only if $\nu_{g}(X)-g$ is a subgroup of $G$. In particular, $X$ is a $C^{0}$-manifold near $g$.

Proof. : If $\nu_{g}(X)-g$ is a subgroup then for every $h \in \nu_{g}(X)$, we have $h-g+\nu_{g}(X)=\nu_{g}(X)$. It easily follows that $X$ is locally $G$-linear at $g$.

Conversely, assume that $X$ is locally $G$-linear at $g$. We need to prove: For all $h_{1}, h_{2} \in X$ sufficiently close to $g$ we have $\left(h_{1}-g\right)-\left(h_{2}-g\right)=h^{\prime}-g$, for some $h^{\prime} \in X$, or equivalently, $g+\left(h_{1}-h_{2}\right) \in X$.

We pick $h \in X$ close to $g$ and generic over $g$ such that $X-g={ }_{0} X-h$. We take $U \ni 0$, definable over parameters independent of $g, h$, such that $(X-g) \cap U=(X-h) \cap U$. Because of the genericity of $h$, there exists a neighborhood $V \ni h$, which we may assume is contained in $h+U$, such that for all $h^{\prime} \in X \cap V$,

$$
(X-h) \cap U=(X-g) \cap U=\left(X-h^{\prime}\right) \cap U .
$$


By adding $h$ to both sides of the last equation, we obtain: For all $h^{\prime} \in X \cap V$, $X \cap(h+U)=\left(X-h^{\prime}+h\right) \cap(h+U)$, hence (since $\left.V \subseteq(h+U)\right)$ also for all $h^{\prime} \in X \cap V$,

$$
X \cap V=\left(X-h^{\prime}+h\right) \cap V .
$$

In particular, if $h^{\prime \prime}, h^{\prime} \in X$ are sufficiently close to $h$ (so that $h^{\prime \prime}-h^{\prime}+h \in$ $V)$ then we have $\left(h^{\prime \prime}-h^{\prime}\right)+h$ in $X$. It follows, as was pointed out above, that $\nu_{h}(X)-h$ is a subgroup of $G$. Because $\nu_{g}(X)-g=\nu_{h}(X)-h$, it also follows that $\nu_{g}(X)-g$ is a subgroup of $G$.

Because the germ of $X$ at $g$ is $G$-equivalent to the germ of $X$ at some generic point near $g$, it follows that $X$ is a $C^{0}$-manifold near $g$.

Notation. For a $G$-linear $X$, we denote by $\nu(X)$ the group $\nu_{x}(X)-x$, for some (all) $x \in X$. Notice that $Y$ is a $G$-subset of $X$ iff $\nu_{h}(Y)-h$ is a subset of the group $\nu(X)$, for every $h \in Y$. Note also that two $G$-linear sets $X_{1}, X_{2}$ are $G$-equivalent if and only if $\nu\left(X_{1}\right)=\nu\left(X_{2}\right)$.

Lemma 2.7. Let $X, Y \subseteq G$ be definable sets of dimension d. If $\operatorname{dim}(X+$ $Y)=d$ then $X_{\text {lin }}$ is large in $X, Y_{\text {lin }}$ large in $Y$ and $X_{\text {lin }}^{\max }, Y_{\text {lin }}^{\max }$ are $G$-linear sets that are $G$-equivalent.

Proof. We assume that $X$ and $Y$ are $\emptyset$-definable and write $Z=X+Y$. Take $g$ generic in $X$ over $\emptyset$ and $h$ generic in $Y$ over $g$. Then $g+h$ is generic in $Z$ over each $g$ and $h$ and hence for every two $x, y \in\{g, h, g+h\}$ we have $\operatorname{dim}(x / y)=d$.

For every $\emptyset$-definable neighborhood $U$ of $g$, we have $(U \cap X)+h \subseteq Z$. Moreover $g+h$ is generic in $(U \cap X)+h$ over $h$ and therefore $(U \cap X)+h$ contains $\nu_{g+h}(Z)$. It follows that $\nu_{g}(X)+h \supseteq \nu_{g+h}(Z)$. By symmetry we may conclude that $\nu_{g}(X)+h=\nu_{g+h}(Z)$, or equivalently, $\nu_{g}(X)-g=$ $\nu_{g+h}(Z)-(g+h)$.

In the very same way we see that $\nu_{h}(Y)-h=\nu_{g+h}(Z)-(g+h)$. Hence, we showed that for every generic $g \in X, h \in Y$ which are independent from each other we have $\nu_{g}(X)-g=\nu_{h}(Y)-h$.

Fixing a generic $h \in Y$, this implies that $\nu_{g^{\prime}}(X)$ is constant as $g^{\prime}$ varies over all elements $g^{\prime}$ which are generic in $X$ over $h$. In particular, $X_{\text {lin }}$ contains all those elements $g^{\prime}$ and moreover all the components of $X_{\text {lin }}$ of dimension $d$ are $G$-equivalent. The same argument shows that all components of $Y_{\text {lin }}$ of dimension $d$ are $G$-equivalent to each other and that $X_{\text {lin }}^{\max }$ is $G$-equivalent to $Y_{\text {lin }}^{\max }$. 
Corollary 2.8. Let $X, Y, Z \subseteq G$ be definable sets of dimension d. Assume that for every $g$ in a large set $X_{0} \subseteq X$, we have $\operatorname{dim}((g+Y) \cap Z)=d$. Then $X_{\text {lin }}$ is large in $X$.

Proof. Without loss of generality, $X, Y, Z, X_{0}$ are $\emptyset$-definable, and hence every generic element $g \in X$ belongs to $X_{0}$. For every such $g \in X_{0}$ there exists $c \in(g+Y) \cap Z$ which is generic in $Z$ over $g$. It follows that $h=c-g$ is in $Y$ and, as before, the dimension of any two of $\{g, h, c\}$ is $2 d$. We can now find open neighborhoods $V$ of $h$ and $W$ of $c$, defined over independent parameters, such that $g+(V \cap Y) \subseteq(W \cap Z)$. Because of the genericity of $g$ there is a neighborhood $U$ of $g$ such that $(U \cap X)+(V \cap Y) \subseteq(W \cap Z)$. In particular, $\operatorname{dim}((U \cap X)+(V \cap Y))=d$. We can therefore apply Lemma 2.7 to $U \cap X$ and $V \cap Y$ and conclude that $X$ is locally $G$-linear at $g$.

Note that we cannot conclude, under the assumption of the last Lemma, that all components of $X_{\text {lin }}^{\max }$ are $G$-equivalent.

Definition 2.9. If $U$ is an open symmetric $(U=-U)$ neighborhood of 0 and $Y \subseteq G$ a definable set, we say that $h_{1}, h_{2} \in Y$ are $U$-connected in $Y$ if $h_{2} \in h_{1}+U$ and there exists a definable path in $Y$ connecting $h_{1}$ and $h_{2}$, which is contained in $h_{1}+U$.

Lemma 2.10. Let $X$ be $G$-linear and $Y$ a $G$-subset of $X$. Take $g \in X$, and assume that $U$ is an open symmetric neighbourhood of 0 such that $(g+U) \cap X$ is relatively closed in $g+U$. Then, for any $h_{1}, h_{2} \in Y$ that are $U$-connected in $Y$, we have $g+\left(h_{1}-h_{2}\right) \in X$

Proof. Because $h_{1}, h_{2}$ are $U$-connected in $Y$, there exists a definable path $\gamma:[a, b] \rightarrow Y$ such that $\gamma(a)=h_{1}, \gamma(b)=h_{2}$ and for every $t \in[a, b]$, $\gamma(t)-h_{1} \in U$. Consider the set $T$ of all $t \in[a, b]$ such that $g+\left(h_{1}-\gamma\left(t^{\prime}\right)\right) \in X$, for all $t^{\prime} \leq t$.

We claim that $T$ is both open and closed (with respect to the order topology in $M)$ in $[a, b]$. Indeed, because $(g+U) \cap X$ is relatively closed in $g+U$, the set $T$ is closed in $[a, b]$.

To see that it is open, assume that $t_{0} \in T$. In particular, $k=g+\left(h_{1}-\right.$ $\left.\gamma\left(t_{0}\right)\right)$ is in $X$. For $t^{\prime} \in[a, b]$ close to $t_{0}$ we have $\left(\gamma\left(t_{0}\right)-\gamma\left(t^{\prime}\right)\right) \in X-k$ (because $\nu_{\gamma\left(t_{0}\right)}(Y)-\gamma\left(t_{0}\right) \subseteq \nu_{k}(X)-k=\nu(X)$ is a subgroup of $G$ ), therefore $t^{\prime} \in T$. It follows that $T$ is open and closed in $[a, b]$, hence $T=[a, b]$ and therefore $g+\left(h_{1}-h_{2}\right) \in X$.

The following technical lemma ensures that we can extend every $G$ linear set and every $G$-subset of a $G$-linear set beyond its frontier (where the frontier of $X$ is $C l(X) \backslash X)$. 
Lemma 2.11. Assume that $X$ is $G$-linear and $Y$ is $G$-subset of $X$. Then $\mathrm{Cl}(Y)$ is also a $G$-subset of $X$.

Proof. Fix $h$ in $C l(Y) \backslash Y$. We first prove that $\nu_{h}(Y)-h \subseteq \nu(X)$. Namely we show: For all $h^{\prime} \in Y$ sufficiently close to $h$ and every $g \in X$ we have $g+h^{\prime}-h \in X$.

If the above fails then we have a curve $\gamma:[a, b) \rightarrow Y$, with $\lim _{t \rightarrow b} \gamma(t)=$ $h$ such that for all $t \in[a, b), g+\gamma(t)-h \notin X$. Fix $U$ an open symmetric neighborhood of 0 such that $X \cap(g+U)$ is relatively closed in $g+U$. By choosing $\gamma(a)$ sufficiently close to $h$, we may assume that for every $t \in[a, b]$, we have $\gamma(t) \in \gamma(a)+U$ (so in particular, $h \in \gamma(a)+U$ ). It follows that for every $t \in(a, b)$, we have $\gamma(a)$ and $\gamma(t)$ are $U$-connected (as witnessed by $\gamma)$ and therefore, by Lemma 2.10, $g+\gamma(a)-\gamma(t) \in(g+U) \cap X$. Because $X \cap(g+U)$ is closed in $g+U$, we may take $t$ to be $b$ and conclude that $g+\gamma(a)-\gamma(b)=g+\gamma(a)-h \in X$, contradicting our assumption.

We therefore showed that for all $h \in C l(Y) \backslash Y, \nu_{h}(Y)-h \subseteq \nu(X)$. By our assumption on $Y$ this is true for every $h \in C l(Y)$. Because $X$ is locally closed we may take the closure on the left and conclude that $\nu_{h}(C l(Y))-h \subseteq \nu(X)$ for every $h \in C l(Y)$, hence $C l(Y)$ is a $G$-subset of $X$.

Lemma 2.12. Let $X \subseteq G$ be a definable set. Assume that

(i) $X_{\text {lin }}$ is large in $X$ (i.e. $\operatorname{dim}\left(X \backslash X_{\text {lin }}\right)<\operatorname{dim}(X)$ ) and

(ii) for every $h \in X$ and $g \in X_{\text {lin }}, X-g \subseteq_{0} X-h$.

Then $X$ is G-linear.

Proof. Assume that $\operatorname{dim}(X)=d$. First notice that by (ii), all the components of $X_{\text {lin }}$ are $G$-equivalent to each other and therefore $X_{l i n}$ is itself a $G$-linear set of dimension $d$.

Because of (ii) the local dimension of $X$ at every point is $d$ and therefore, by (i), $X_{\text {lin }}$ is dense in $X$. By Lemma 2.11, $X$ is a $G$-subset of $X_{l i n}$.

Given any $h \in X$, we have

$$
\nu_{h}(X)-h \subseteq \nu\left(X_{\text {lin }}\right) \subseteq \nu_{h}(X)-h .
$$

(the left inclusion follows from the fact that $X$ is a $G$-subset of $X_{l i n}$ while the right one is just assumption (ii)).

It follows that $X$ is locally $G$-linear at $h$ hence $X$ is $G$-linear.

Before the next lemma we make a small observation.

Remark 2.13. Let $Z, W \subseteq G$ be definable sets and let $f: Z \rightarrow W$ be a definable continuous map (all are 0 -definable). Then for every $w \in f(Z)$ that 
is locally generic in $W$ and $z \in f^{-1}(w)$, we have $\nu_{w}(W)=f\left(\nu_{z}(Z)\right)$. (By "locally generic" we mean that for some open $V \ni w$, we have $\operatorname{dim}(w / \emptyset)=$ $\operatorname{dim}(V \cap W))$.

Proof. Fix $V$ as above and let $U \subseteq G$ be a definable open neighborhood of $z$ such that $w$ is still generic in $V \cap W$ over the parameters (say $A$ ) defining $U$. Then $\operatorname{dim}(w / A)=\operatorname{dim}(w)=\operatorname{dim} f(U \cap Z), w \in f(U \cap Z)$ and therefore $f(U \cap Z)$ and $V \cap W$ have the same germ at $w$. In particular, $\nu_{w}(W)=$ $\nu_{w}(V \cap W) \subseteq f(U \cap Z)$. Because this is true for every $U$ neighborhood of $z$, it follows that $\nu_{w}(W) \subseteq f\left(\nu_{z}(Z)\right)$. The converse follows by continuity.

Lemma 2.14. Let $X$ and $Y$ be two G-linear sets. Then:

(i) $X+Y$ is G-linear and we have

$$
\nu(X+Y)=\nu(X)+\nu(Y) .
$$

In particular, if $X$ and $Y$ are $G$-equivalent $G$-linear sets then $X+Y$ is $G$-equivalent to them as well.

(ii) If $X$ and $Y$ are $G$-equivalent then $X \cup Y$ is a $G$-linear set and $G$ equivalent to $X$ and $Y$.

Proof. (i) First, notice that, by continuity, for any $x \in X, y \in Y$, and $z=x+y$ we have $\nu_{x}(X)+\nu_{y}(Y) \subseteq \nu_{z}(X+Y)$. It follows that for all $z \in X+Y$, we have $\nu(X)+\nu(Y) \subseteq \nu_{z}(X+Y)-z$.

By Remark 2.13, if $z=x+y$ is a generic element of $X+Y$ then $\nu_{z}(X+$ $Y)-z=\nu_{x}(X)+\nu_{y}(Y)-z=\nu(X)+\nu(Y)$. Therefore, $X+Y$ is $G$-linear at every generic $z \in X+Y$ and we have $\nu_{z}(X+Y)-z=\nu(X)+\nu(Y)$.

It follows that $(X+Y)_{\text {lin }}$ is large in $X+Y$ and the germs of $X+Y$ at all points in $(X+Y)_{l i n}$ are $G$-equivalent. Taken together with the above, we see that $X+Y$ satisfies the assumptions of Lemma 2.12(1), hence it is $G$-linear and we have $\nu(X+Y)=\nu(X)+\nu(Y)$.

If $X$ and $Y$ are $G$-equivalent then $\nu(X)=\nu(Y)$, hence $\nu(X)+\nu(Y)=$ $\nu(X)$, so $X+Y$ is $G$-equivalent to both $X$ and $Y$.

(ii) It is easy to see that for all $z \in X \cup Y$, if $x \notin \operatorname{Fr}(X) \cup \operatorname{Fr}(Y)$, then we have $(X \cup Y)-z=_{0} X-z$ (if $\left.z \in X\right)$ or $(X \cup Y)-z=_{0} Y-z$ (if $z \in Y$ ). Because $X$ and $Y$ are $G$-linear and $G$-equivalent, it follows that $(X \cup Y)_{l i n}$ is large in $X \cup Y$. Also, for every $x \in X$ we clearly have $X-x \subseteq_{0}(X \cup Y)-x$, and similarly for $y \in Y$. We therefore can apply Lemma 2.12(1) again and conclude that $X \cup Y$ is $G$-linear, and $G$-equivalent to $X$ and $Y$.

Lemma 2.15. Let $X$ be a $G$-linear set and $Y_{1}, Y_{2}$ two $G$-subsets of $X$. Then (i) $Y_{1}+Y_{2}$ is a $G$-subset of $X$.

(ii) $Y_{1} \cup Y_{2}$ is a $G$-subset of $X$. 
Proof. (i) Because $Y_{1}$ and $Y_{2}$ are $G$-subsets of $X$, for all $y_{1} \in Y_{1}, y_{2} \in Y_{2}$, we have

$\left(\nu_{y_{1}}\left(Y_{1}\right)+\nu_{y_{2}}\left(Y_{2}\right)\right)-\left(y_{1}+y_{2}\right)=\left(\nu_{y_{1}}\left(Y_{1}\right)-y_{1}\right)+\left(\nu_{y_{2}}\left(Y_{2}\right)-y_{2}\right) \subseteq \nu(X)+\nu(X)=\nu(X)$.

Again as before, if $z=y_{1}+y_{2}$ is a locally generic element of $Y_{1}+Y_{2}$ then

$$
\nu_{z}\left(Y_{1}+Y_{2}\right)-z=\nu_{y_{1}}\left(Y_{1}\right)+\nu_{y_{2}}\left(Y_{2}\right)-z \subseteq \nu(X)
$$

and hence $Y_{1}+Y_{2}$ is $G$-subset of $X$ at $z$. We thus have $\left(Y_{1}+Y_{2}\right)_{s l i n}$ dense in $Y_{1}+Y_{2}$. By Lemma 2.11, $Y_{1}+Y_{2}$ is a $G$-subset of $X$.

(ii) Here we only need to note that for $z \in Y_{1} \cap Y_{2}$, it is not true in general that the germs of $Y_{1}$ and $Y_{2}$ at $z$ coincide. However, it is still true that $\left(Y_{1} \cup Y_{2}\right)-z \subseteq_{0} X-g$, so we can proceed as before.

We recall the following definition:

Definition 2.16. Given a definable group $G$ in a sufficiently saturated $\mathcal{M}$, a subgroup $\mathcal{H}$ of $G$ is called locally-definable, if it can be written as the directed union of definable sets $\mathcal{H}=\bigcup\left\{X_{i}: i \in I\right\}$, where $|I|<\kappa$.

$W e$ say that $\mathcal{H}$ is definably connected if the $X_{i}$ 's can all be chosen to be definably connected.

Such groups were sometimes called $\bigvee$-definable groups (see [6]) or Inddefinable groups (see [4]). The dimension of a locally-definable group is taken to be $\max \left\{\operatorname{dim} X_{i}: i \in I\right\}$. Notice that if $\mathcal{H}$ is definably connected then it is actually definably path connected in the sense that any two points can be connected by a definable path in $\mathcal{H}$. The following claim is easy to verify:

Claim 2.17. If $\mathcal{H}=\bigcup\left\{X_{i}: i \in I\right\}$ is a locally-definable group and $g \in \mathcal{H}$ then there exists $i \in I$ such that $g \in X_{i}$ and

$$
\nu_{g}(\mathcal{H})=g+\nu_{0}(\mathcal{H})=\nu_{g}\left(X_{i}\right)
$$

Lemma 2.18. Every locally-definable subgroup $\mathcal{H}$ of $G$ can be written as the directed union of $G$-linear sets.

Proof. Without loss of generality all $X_{i}$ 's in the definition of $\mathcal{H}$ have maximal dimension $d$. By Claim 2.17, for every $g \in \mathcal{H}$ there exists $i \in I$ such that $\nu_{g}(\mathcal{H})=\nu_{g}\left(X_{i}\right)$. For such an $i$ we have: $g \in\left(X_{i}\right)_{l i n}$, the local dimension of $X_{i}$ at $g$ equals $d$ (hence, $\left.g \in\left(X_{i}\right)_{\text {lin }}^{\max }\right)$ and all $\left(X_{i}\right)_{\text {lin }}^{\max }$ are $G$-linear and $G$-equivalent. If we let $X_{i}^{\prime}=\left(X_{i}\right)_{\text {lin }}^{\max }$ then we have $\mathcal{H}=\bigcup_{i \in I} X_{i}^{\prime}$. 
To see that $\left\{X_{i}^{\prime}: i \in I\right\}$ is a directed system of sets: Given $i, j \in I$, we take $k \in I$ such that $X_{i} \cup X_{j} \subseteq X_{k}$ and claim that $X_{i}^{\prime} \cup X_{j}^{\prime} \subseteq X_{k}^{\prime}$. Indeed, if $g \in X_{i}^{\prime}$ then $\nu_{g}\left(X_{i}^{\prime}\right)=\nu_{g}(\mathcal{H})$ and hence $\nu_{g}\left(X_{i}\right)=\nu_{g}\left(X_{k}\right)$. In particular, $g \in\left(X_{k}\right)_{l i n}^{\max }=X_{k}^{\prime}$.

Lemma 2.19. We assume $\mathcal{M}$ is an w-saturated structure. Let $X$ be a definable $G$-linear set, $0 \in X$. Then the following hold.

(1) The group $\langle X\rangle$ generated by $X$ is locally-definable and its germ at 0 equals to $\nu(X)$. In particular, $\operatorname{dim}(\langle X\rangle)=\operatorname{dim} X$.

(2) If $Y$ a $G$-subset of $X$ containing 0 , then the group generated by $Y$ is a locally-definable of dimension $\leq \operatorname{dim} X$, whose germ at 0 is contained in $\nu(X)$.

Proof. (1) The group $\langle X\rangle$ generated by $X$ is a countable increasing union of the sets $X_{0}=X, X_{1}=X-X, X_{2}=(X-X)+(X-X), \ldots$, so locallydefinable. By the Lemma 2.14, each $X_{n}$ is $G$-linear and $G$-equivalent to $X$ (so in particular, has the same dimension as $X$ ). Given $g \in\langle X\rangle$, there exists $n \geq 0$ such that $g \in X_{n}$. Because of the $G$-linearity, for every $k \geq n$, we have $X_{n}={ }_{g} X_{k}$. Because of saturation, there exists a neighborhood $U$ of $g$ such that $U \cap\langle X\rangle=U \cap X_{n}$. It follows that the germ of $\langle X\rangle$ at this point equals to that of $X_{n}$ and in particular, $\langle X\rangle_{0}=\nu(X)$.

(2) The group $\langle Y\rangle$ generated by $Y$ is a countable increasing union of the sets $Y_{0}=Y, Y_{1}=Y-Y, Y_{2}=(Y-Y)+(Y-Y), \ldots$ Because $-Y$ is also a $G$-subset of $X$, we can apply Lemma 2.15 and conclude that each $Y_{n}$ is a $G$-subset of $X$ whose germ at 0 is contained in $\nu(X)$. It follows that the dimension of $\langle Y\rangle$ is at most $\operatorname{dim} X$ and that the germ of $\langle Y\rangle$ at 0 is contained in that of $\langle X\rangle$.

We end this section with a small observation on locally-definable subgroups:

Lemma 2.20. Let $\mathcal{H}_{1}, \mathcal{H}_{2}$ be locally-definable subgroups of $G$. Then $\mathcal{H}_{1}+\mathcal{H}_{2}$ is a locally-definable group whose germ at 0 equals to the sum of the germs of $\mathcal{H}_{1}$ and $\mathcal{H}_{2}$ at 0.

Proof. Let

$$
\mathcal{H}_{1}=\bigcup_{i \in I} X_{i} ; \mathcal{H}_{2}=\bigcup_{j \in J} Y_{j}
$$

As was pointed out above, we may assume that the $X_{i}$ 's $Y_{j}$ 's are all $G$ linear. By Lemma 2.14(1), the sets $X_{i}+Y_{j}$ are all $G$-linear and $\nu\left(X_{i}+Y_{j}\right)=$ $\nu\left(X_{i}\right)+\nu\left(Y_{j}\right)$. By Lemma 2.19(1),

$$
\nu_{0}\left(\mathcal{H}_{1}+\mathcal{H}_{2}\right)=\nu\left(X_{i}+Y_{j}\right)=\nu\left(X_{i}\right)+\nu\left(Y_{j}\right)=\nu_{0}\left(\mathcal{H}_{1}\right)+\nu_{0}\left(\mathcal{H}_{2}\right) .
$$




\section{Definable sets containing abstract subgroups}

In this section $\mathcal{M}$ will denote an $\omega$-saturated o-minimal expansion of an ordered group.

Theorem 3.1. Let $G$ be a definable abelian group, $\Gamma \subseteq G$ an abstract subgroup (i.e., $\Gamma$ is not necessarily definable). Let $X \subseteq G$ be a definable set containing $\Gamma$ of minimal dimension $d$. Then there exist a definable set $X^{\prime}$ of dimension $d$, a definably connected locally-definable subgroup $\mathcal{H}$ of dimension $d$, and $g_{1}, \ldots, g_{k} \in \Gamma$ such that $\Gamma \subseteq X^{\prime} \subseteq \bigcup_{i=1}^{k} \mathcal{H}+g_{i}$.

Proof. Assume the nontrivial case $d>0$. For every $g \in \Gamma$, the set $X \cap(g+X)$ contains $\Gamma$ and, hence by minimality, has dimension $d$. It follows that the set $Y=\{g \in X: \operatorname{dim}(X \cap X+g)=d\}$ contains $\Gamma$ and therefore, again by minimality, has dimension $d$.

We now apply Lemma 2.8 to $Y, Y, X$ and $X$ (for $X_{0}, X, Y$ and $Z$, respectively), and conclude that $Y_{\text {lin }}$ is large in $Y$.

Since $Y$ contains $\Gamma$ we may replace $X$ with $Y$ and assume from now on that $X_{l i n}$ is large in $X$ (however, it need not be the case that $X$ or even $X_{\text {lin }}$ is $G$-linear). Moreover, we pick $X \supseteq \Gamma$ such that $X_{l i n}$ has the minimal number of definably connected components of dimension $d$. Note that each component has infinitely many elements of $\Gamma$ (otherwise, we can replace it by finitely many points).

Claim 1 All the components of $X_{\text {lin }}$ of dimension $d$ are $G$-equivalent to each other.

Proof Indeed, if $X_{1}, X_{2}$ are two such components then for $g \in X_{1} \cap \Gamma$, the set $\left\{h \in X_{2}: g+h \in X\right\}=X_{2} \cap X-g$ contains $X_{2} \cap \Gamma$ and therefore has dimension $d$ (otherwise, we could replace $X_{2}$ by a definable set of smaller dimension). For the same reason, the set of all $g \in X_{1}$ such that $\operatorname{dim}\left(X_{2} \cap(X-g)\right)=d$ has dimension $d$. Just like in the proof of Corollary 2.8, we can apply Lemma 2.7 locally to $X_{1}$ and $X_{2}$, and conclude that for some open $U$ and $V$ we have $U \cap X_{1}$ is $G$-equivalent to $V \cap X_{2}$. Because $X_{1}$ and $X_{2}$ are $G$-linear it follows that they are $G$-equivalent to each other. End of Claim 1.

We therefore showed that $X_{\text {lin }}^{\max }$ is $G$-linear. It is left to handle $X^{*}=$ $X \backslash X_{\text {lin }}^{\max }$. Fix one of the components $X_{0}$ of $X_{\text {lin }}^{\max }$.

As before, for every $g \in \Gamma \cap X^{*}$, the set $\left\{h \in X_{0}: g+h \in X\right\}$ contains $X_{0} \cap \Gamma$ and hence has dimension $d$. Therefore, after possibly replacing $X^{*}$ 
by a smaller set we may assume that for all $g \in X^{*}$ the set $\left(g+X_{0}\right) \cap X$ has dimension $d$.

Claim $2 X$ is a $G$-subset of $X_{\text {lin }}^{\max }$.

Proof By abuse of notation we let $\nu(X)$ be the infinitesimal subgroup associated to the $G$-linear set $X_{\text {lin }}^{\max }$. By Lemma 2.11, it is enough to see that a dense subset of $X$ is $G$-linear in $X_{\text {lin }}^{\max }$. Namely, we will show that for every locally generic $g \in X$, we have $\nu_{g}(X)-g \subseteq \nu(X)$. It is clearly sufficient to consider $g \in X^{*}$.

We fix an open set $U \subseteq G$ and $g \in X^{*}$ which is generic in $U \cap X$. By our assumption on $X^{*}$, there exists $h$ generic in $X_{0}$ over $g$ such that $g+h$ (generic) in $X$. As in the proof of Lemma 2.7, it follows that $\nu_{g}(X)+h \subseteq$ $\nu_{g+h}(X)$, hence

$$
\nu_{g}(X)-g \subseteq \nu_{g+h}(X)-(g+h)=\nu(X) .
$$

(the right-most equality follows from the fact that $g+h$ is generic in $X$ and hence belongs to $X_{\text {lin }}^{\max }$ ). End of Claim 2.

Let $X_{\text {lin }}^{\max }=X_{1} \cup \cdots \cup X_{r}$ be the union of those components of $X_{\text {lin }}$ of dimension $d$. By Lemma 2.4 each $X_{i}$ is $G$-linear. For each $X_{i}$, pick $g_{i} \in \Gamma \cap X_{i}$, and consider the set $X_{i}^{\prime}=X_{i}-g_{i}$. By Claim 1, the union $X^{\prime}=\bigcup_{i=1}^{r} X_{i}^{\prime}$ is $G$-linear (and $\nu\left(X^{\prime}\right)=\nu\left(X_{i}\right)$ for every $i$ ). It also contains 0 and it is definably connected. Let $\mathcal{H}^{\prime}$ be the subgroup of $G$ generated by $X^{\prime}$. By Lemma 2.19(1), $\operatorname{dim} \mathcal{H}^{\prime}=\operatorname{dim} X^{\prime}=d$. We thus have

$$
X_{\text {lin }}^{\max } \subseteq \bigcup_{i=1}^{r} \mathcal{H}^{\prime}+g_{i} .
$$

Let $X^{1}, \ldots, X^{t}$ be the definably connected components of $X^{*}$. For every such $X^{j}$, we may assume that $X^{j} \cap \Gamma \neq \emptyset$ (for otherwise we may omit this component), take $g^{j} \in \Gamma \cap X^{j}$, and let $X^{\prime \prime}=\bigcup_{i=1}^{t} X^{j}-g^{j}$. By Claim 2, each $X^{j}$ (and therefore also $X^{j}-g^{j}$ ) is a $G$-subset of $X_{\text {lin }}^{d}$. By Lemma 2.15 (2), the set $X^{\prime \prime}$ is also a $G$-subset of $X_{\text {lin }}^{\max }$. It is also definably connected and contains 0 .

Let $\mathcal{H}^{\prime \prime}$ be the subgroup of $G$ generated by $X^{\prime \prime}$. By Lemma 2.19(2), the germ of $\mathcal{H}^{\prime \prime}$ at 0 is contained in that of $X^{\prime}$ and therefore of $\mathcal{H}^{\prime}$. We now let $\mathcal{H}=\mathcal{H}^{\prime}+\mathcal{H}^{\prime \prime}$. By Lemma 2.20, we have $\nu_{0}(\mathcal{H})=\nu_{0}\left(\mathcal{H}^{\prime}+\mathcal{H}^{\prime \prime}\right)=\nu_{0}\left(\mathcal{H}^{\prime}\right)$, so in particular the dimension of $\mathcal{H}$ is $d$.

Putting the above facts together we obtain:

$$
\Gamma \subseteq X \subseteq \bigcup_{i=1}^{k} \mathcal{H}+g_{i}
$$


for some $g_{1}, \ldots, g_{k} \in \Gamma$.

With that we end the proof of the theorem.

Corollary 3.2. Let $G$ be a definably connected abelian group. Let $\Gamma$ be a divisible subgroup of the subgroup of torsion points Tor $(G)$. Let $X \subset G$ be a definable set containing $\Gamma$. Then, there is a definably connected locallydefinable $\mathcal{H}$ subgroup of $G$ with $\operatorname{dim} \mathcal{H} \leq \operatorname{dim} X$ such that $\Gamma \subset \mathcal{H}$.

Proof. By Theorem 3.1, taking $X$ of minimal dimension, there is a definably connected locally-definable group $\mathcal{H}$ subgroup of $G$, with $\operatorname{dim} \mathcal{H}=\operatorname{dim} X$, and $g_{1}, \ldots, g_{k} \in \Gamma$ such that $\Gamma \subseteq \bigcup_{i=1}^{k} \mathcal{H}+g_{i}$. Then $\Gamma \subset \mathcal{H}$. Indeed, let $g \in \Gamma$ and let $m=l c m\left(o\left(g_{1}\right), \ldots, o\left(g_{k}\right)\right)$, since $\Gamma$ is divisible there is $h \in \Gamma$ such that $g=m h$. For such $h$ there is an $i$ such that $h=h^{\prime}+g_{i}$, for some $h^{\prime} \in \mathcal{H}$. Then $g=m h=m h^{\prime} \in \mathcal{H}$.

\section{The main result: commutative case}

In this section $\mathcal{M}$ will be an $\omega$-saturated o-minimal expansion of a real closed field and $G$ will denote a definably compact definably connected abelian group of dimension $n$. Such $G$ is divisible and hence the subgroup $\operatorname{Tor}(G)$ and any $p$-Sylow of $G$ are also divisible (the $p$-Sylow of $G$ is $G_{p}=\bigcup_{n>0} G\left[p^{n}\right]$, where $\left.G[m]=\left\{g \in G: m g=0_{G}\right\}\right)$.

By Theorem 1.1 in $[3]$ we have $G[m] \cong(\mathbb{Z} / m \mathbb{Z})^{n}$ for any $m>0$, and $\pi_{1}(G) \cong \mathbb{Z}^{n}$, where $\pi_{1}(G)$ is the $o$-minimal fundamental group of $G$.

Let $p$ be a prime number and let $x_{1}, \ldots, x_{n} \in G[p]$ we say that $x_{1}, \ldots, x_{n}$ are $n$ independent $p$-torsion points if they are $\mathbb{F}_{p}$-independent under the isomorphism $G[p] \cong \mathbb{F}_{p}{ }^{n}$.

Theorem 4.1. Let $X$ be a definable subset of $G$. If $X$ contains a $p$-Sylow of $G$, then $\operatorname{dim} X=n$.

Lemma 4.2. Let $p$ be a prime number and let $x_{1}, \ldots, x_{n} \in G$ be $n$ independent p-torsion points. For each $i=1, \ldots, n$ let $\tau_{i}$ be a path in $G$ from $0_{G}$ to $x_{i}$, and let $p \tau_{i}$ denote the loop at $0_{G}, t \mapsto p \tau_{i}(t)$. Then, $\left[p \tau_{1}\right], \ldots,\left[p \tau_{n}\right] \in \pi_{1}(G)$ are $\mathbb{Z}$-linearly independent.

Proof. Let $\varphi: \pi_{1}(G) \rightarrow G[p]$ the homomorphism $[\gamma] \mapsto \tilde{\gamma}(1)$, where $\tilde{\gamma}$ is the unique path in $G$ starting at $0_{G}$ and such that $p \tilde{\gamma}=\gamma$ (see the proofs of propositions 2.10 and 2.11 in [3]). Suppose $\left[p \tau_{1}\right], \ldots,\left[p \tau_{n}\right]$ are $\mathbb{Z}$-linearly dependent and let $m_{1}\left[p \tau_{1}\right]+\cdots+m_{n}\left[p \tau_{n}\right]=\left[k_{0_{G}}\right]$ with $\left(m_{1}, \ldots, m_{n}\right)=1$, where $k_{0_{G}}$ is the constant loop at $0_{G}$. Applying $\varphi$ to this equality we get $m_{1} x_{1}+\cdots+m_{n} x_{n}=0_{G}$ and hence $p \mid\left(m_{1}, \ldots, m_{n}\right)$, a contradiction. 
Lemma 4.3. Let $\left[\gamma_{1}\right], \ldots,\left[\gamma_{n}\right] \in \pi_{1}(G)$ be $\mathbb{Z}$-linearly independent. Then,

$$
\left\{\sum_{i=1}^{n} \gamma_{i}\left(t_{i}\right): t_{i} \in[0,1), 1 \leq i \leq n\right\}=G .
$$

Proof. Consider the definably compact definably connected $n$-dimensional abelian group $\mathbb{T}=[0,1)^{n}$ and the definable map

$$
\begin{array}{cccc}
f: & \mathbb{T} & \longrightarrow & G \\
& \left(t_{1}, \ldots, t_{n}\right) & \mapsto & \sum_{i=1}^{n} \gamma_{i}\left(t_{i}\right) .
\end{array}
$$

It suffices to prove that $f$ is onto. Since $f$ is continuous with respect the manifold topology of $\mathbb{T}$, we can see $f$ as a definable continuous map between definable manifolds.

Claim It suffices to prove that $f$ induces an isomorphism $f_{*}: H_{1}(\mathbb{T} ; \mathbb{Q}) \rightarrow$ $H_{1}(G ; \mathbb{Q})$.

Proof The map $f$ has a degree for any orientations of $\mathbb{T}$ and $G$, since both $\mathbb{T}$ and $G$ have dimension $n$ (see section 4 in [3]). To prove that $f$ is onto it suffices to prove that one (equivalently each one) of these degrees is not zero. Let $\zeta_{G}$ and $\zeta_{\mathbb{T}}$ be the fundamental classes of some given orientations of $G$ and $\mathbb{T}$, i.e., $\zeta_{G}$ (respectively $\zeta_{\mathbb{T}}$ ) is a generator of $H_{n}(G) \cong \mathbb{Z}$ (resp. of $\left.H_{n}(\mathbb{T}) \cong \mathbb{Z}\right)$. The degree of $f$ is defined by $f_{*}\left(\zeta_{\mathbb{T}}\right)=\operatorname{deg}(f) \zeta_{G}$. If $\omega_{G}$ and $\omega_{\mathbb{T}}$ are the corresponding cohomology classes by duality we have $f^{*}\left(\omega_{G}\right)=\operatorname{deg}(f) \omega_{\mathbb{T}}$. Hence to prove that $\operatorname{deg}(f) \neq 0$ suffices to prove that $f$ induces an isomorphism of the $\mathbb{Q}$-vector spaces $f^{*}: H^{n}(G ; \mathbb{Q}) \rightarrow H^{n}(\mathbb{T} ; \mathbb{Q})$. Now $f^{*}: H^{*}(G ; \mathbb{Q}) \rightarrow H^{*}(\mathbb{T} ; \mathbb{Q})$ is also $\mathbb{Q}$-algebra homomorphism and by Theorem 1.1 in $[3]$, both $\mathbb{Q}$-algebras are generated by elements of degree one. So it suffices to prove that $f$ induces an isomorphism $f^{*}: H^{1}(G ; \mathbb{Q}) \rightarrow$ $H^{1}(\mathbb{T} ; \mathbb{Q})$. Applying duality again we have the required result. End of Claim.

Now, let $\delta_{i}:[0,1] \rightarrow \mathbb{T}: t \mapsto \delta_{i}(t)=(0, \ldots, 1, \ldots, 0)$. The map $f$ induces a map $f_{*}: \pi_{1}(\mathbb{T}) \rightarrow \pi_{1}(G):\left[\delta_{i}\right] \mapsto f_{*}\left(\left[\delta_{i}\right]\right)=\left[\gamma_{i}\right]$, for each $i=1, \ldots, n$, which is one to one and has finite cokernel $\left(=\pi_{1}(G) / \operatorname{Im}\left(f_{*}\right)\right)$ because we have $n \mathbb{Z}$-linear independent $\left[\gamma_{i}\right]$ 's. Identifying the $\pi_{1}$ 's with the $H_{1}$ 's via the Hurewicz isomorphism, we have the following exact sequence

$$
0 \rightarrow H_{1}(\mathbb{T}) \stackrel{f_{*}}{\rightarrow} H_{1}(G) \rightarrow H_{1}(G) / \operatorname{Im}\left(f_{*}\right) \rightarrow 0 .
$$

tensoring with $\mathbb{Q}$ we obtain that $f_{*}: H_{1}(\mathbb{T} ; \mathbb{Q}) \rightarrow H_{1}(G ; \mathbb{Q})$ is an isomorphism.

Corollary 4.4. Let $\mathcal{H}$ be a definably connected locally-definable subgroup of $G$. Suppose $\mathcal{H}$ contains $G[p]$, for some prime $p$. Then $\mathcal{H}=G$. 
Proof. Let $x_{1}, \ldots, x_{n}$ be $n$ independent $p$-torsion points and for each $i=$ $1, \ldots, n$ let $\tau_{i}$ be a path in $\mathcal{H}$ from $0_{G}$ to $x_{i}$. Let $\gamma_{i}$ denote the loop at $0_{G}$ defined by $t \mapsto p \tau_{i}(t)$, since $\mathcal{H}$ is a group $\gamma_{i}([0,1]) \subset \mathcal{H}$. By Lemma 4.2 $\left[\gamma_{1}\right], \ldots,\left[\gamma_{n}\right] \in \pi_{1}(G)$ are $\mathbb{Z}$-linearly independent.

By Lemma 4.3, $S=\left\{\sum_{i=1}^{n} \gamma_{i}\left(t_{i}\right): t_{i} \in[0,1), 1 \leq i \leq n\right\}=G$. Again since $\mathcal{H}$ is a group $S \subset \mathcal{H}$.

Proof of Theorem 4.1. Let $X \subseteq G$ be a definable set containing a $p$-Sylow $G_{p}$ of $G$. Since $G_{p}$ is divisible, by Corollary 3.2 , there exists a definably connected locally-definable group $\mathcal{H}$ of dimension $\leq \operatorname{dim} X$ containing $G_{p}$. Since $\mathbb{G}_{p}$ contains $G[p]$, we can apply Corollary 4.4 to get $\mathcal{H}=G$ and hence $\operatorname{dim} X=\operatorname{dim} G$

Corollary 4.5. Let $X$ be a definable subset of $G$. If $X$ is large in $G$ then for any prime $p$ and any $l$ there is an $m>l$ and $a g \in X$ of order $p^{m}$.

Proof. Note that this corollary is equivalent to Theorem 4.1. Indeed, for the nontrivial case suppose there is a prime $p$ and an $l$ such that $X \cap G_{p} \subset G\left[p^{l}\right]$. So the set $X^{\prime}=X \backslash G\left[p^{l}\right]$ is still large in $G$ and $X^{\prime} \cap G_{p}=\emptyset$. But then $G \backslash X^{\prime}$ contains $G_{p}$ and $\operatorname{dim}\left(G \backslash X^{\prime}\right)<\operatorname{dim} G$, a contradiction with the theorem.

\section{The main result: general case}

We work in an $\omega$-saturated structure $\mathcal{M}$ which is an o-minimal expansion of a real closed field $\mathcal{M}$. We will use multiplicative notation for groups, and denote by $p_{k}$ the map $x \mapsto x^{k}$. A definably connected group which is either abelian or definably compact is divisible (see [1] or [2]), hence the map $p_{k}$ is onto. Recall that a generic subset $Y$ of a definable abelian group $G$ is a definable subset of $G$ such that finitely many translates of $Y$ cover $G$.

Lemma 5.1. Let $T$ be a definable abelian group. Suppose that for every $Y \subseteq T$ generic, $Y \cap \operatorname{Tor}(T) \neq \emptyset$. Then, for each $Y \subseteq T$ generic there is a $k \in \mathbb{N}$ such that $p_{k}(Y)=T$.

Proof. First note that for each $Y \subseteq T$ generic, there are $g_{1}, \ldots, g_{l} \in \operatorname{Tor}(T)$ such that $T=\bigcup_{i=1}^{n} g_{i} Y$. Indeed, if not, by compactness, there is $h \notin$ $\operatorname{Tor}(T) Y$, i.e., $h Y^{-1} \cap \operatorname{Tor}(T)=\emptyset$, but $h Y^{-1}$ is also generic, a contradiction. Now taking $k=\operatorname{lcm}\left(o\left(g_{1}\right), \ldots, o\left(g_{l}\right)\right)$ we have $p_{k}(Y)=p_{k}(T)=T$ as required. 
We recall that a maximal definably connected abelian subgroup $T$ of a definably connected definably compact group $G$ is called a maximal torus of $G$ and that $G=\bigcup_{g \in G} T^{g}$ (see Theorem 6.12 in [1] or [2]).

Lemma 5.2. Let $G$ be a definably compact definably connected group. Let $T$ be a maximal torus of $G$ and $X \subseteq G$ is definable. Assume the following:

(i) For every $g \in G$ and for every $Y \subseteq T^{g}$ generic, $Y \cap \operatorname{Tor}\left(T^{g}\right) \neq \emptyset$, and

(ii) For every $g \in G, X \cap T^{g}$ is generic in $T^{g}$.

Then there is a $k \in \mathbb{N}$ such that $p_{k}(X)=G$.

Proof. Given $g \in G$, by (ii), we can apply Lemma 5.1, for $Y=X \cap T^{g}$, to get a $k(g) \in \mathbb{N}$ such that the map $y \mapsto y^{k(g)}$ sends $X \cap T^{g}$ onto $T^{g}$ (note that by divisibility of $T^{g}$ any multiple of $k(g)$ has the same property). Then, by compactness, there is $k \in \mathbb{N}$ such that for every $g \in G$, the map $y \mapsto y^{k}$ sends $X \cap T^{g}$ onto $T^{g}$. To finish the proof suffices to make use of the equality $G=\bigcup_{g \in G} T^{g}$.

For the next lemma we will use the notions of compact domination and very good reduction introduced in [4]. Recall that a definably simple group has very good reduction and that a definably compact group which has very good reduction has also compact domination (see Theorem 10.7 in [4]).

Lemma 5.3. Let $G$ be a definably compact definably connected group. Let $X \subseteq G$ be a definable set such that Tor $(G) \subseteq X$. If $G$ has very good reduction then there is $k \in \mathbb{N}$ such that $p_{k}(X)=G$.

Moreover, if $N$ is a finite (central) normal subgroup of $G$ and $G / N$ has very good reduction then there is $k \in \mathbb{N}$ such that $p_{k}(X)=G$.

Proof. The result can probably be read off standard Lie theory. However, since we could not find a reference we give a complete proof.

Without lost of generality we may assume that $G=G(M)$ is defined over the reals. By our assumptions on $G, G(\mathbb{R})$ is a compact connected Lie group. Let $T_{0}$ be a standard maximal torus of $G(\mathbb{R})$, hence $T_{0}$ is also a Lie group and hence definable over the reals. Let $T=T_{0}(\mathcal{M})$. So $T$ is still a maximal torus of $G$ and it has very good reduction and hence it is compactly dominated. The same happens for every conjugate $T^{g}$ of $T$, for $g \in G$. Since all $T^{g}$ 's are also abelian, we can apply Proposition 10.6 in [4], to get that for every $g \in G$, every generic subset of $T^{g}$ has a torsion point, so condition (i) of Lemma 5.2 is satisfied. 
Because $\operatorname{Tor}(G) \subseteq X$, the set $T^{g} \backslash X$ has no torsion and hence, by (i), it is non-generic. It follows that $T^{g} \cap X$ is generic and therefore (ii) holds. Now we can apply Lemma 5.2 to get the required result.

For the moreover clause, let $\pi: G \rightarrow G / N$ be the projection map. Because $N$ is finite, all torsion elements of $G / N$ are in $\pi(X)$, hence by what we have just proved, there is $k$ such that $p_{k}(\pi(X))=G / N$. If we take $k^{\prime}=k \cdot|N|$ then $p_{k^{\prime}}(X)=G$ (because $N$ is central and $G$ divisible).

Notice, in the setting of the above lemma, that if $p_{k}(X)=G$ then in particular, $\operatorname{dim} X=\operatorname{dim} G$ (since the image of $X$ under a definable map cannot increase).

We can now prove the main result:

Theorem 5.4. Let $G$ be a definably compact group and assume that $X \subseteq G$ is a definable set containing all torsion points of $G$. Then $\operatorname{dim} X=\operatorname{dim} G$.

Proof. By 4.1 in [5] and 5.4 in [8], after modding out $G_{1}=G / Z(G)$ by its finite center, the group we obtain is a direct product of simple groups. In particular, it has very good reduction (see the proof of 5.1 in [7]) and hence the "moreover" clause of Lemma 5.3 holds for $G / Z(G)$. We therefore have:

(i) For every definable $X_{1} \subseteq Z(G)$, if $\operatorname{Tor}(Z(G)) \subseteq X_{1}$ then $\operatorname{dim} X_{1}=$ $\operatorname{dim} Z(G)$ (by Theorem 4.1 this is true for subsets of $Z(G)^{0}$, but then it clearly follows for subsets of $Z(G)$ as well).

(ii) For every definable $X_{1} \subseteq G$, if $\operatorname{Tor}(G / Z(G)) \subseteq X_{1} / Z(G)$ then $\operatorname{dim}\left(X_{1} / Z(G)\right)=\operatorname{dim}(G / Z(G))$. (by Lemma 5.3 and our previous observation).

We now proceed as follows, with $X \subseteq G$ a definable set containing $\operatorname{Tor}(G)$. Given $g \in \operatorname{Tor}(G)$ and $h \in \operatorname{Tor}(Z(G))$, we have $g h \in \operatorname{Tor}(G) \subseteq X$. Hence, for every such $g$, we have

$$
X_{g}=\{h \in Z(G): g h \in X\} \supseteq \operatorname{Tor}(Z(G)),
$$

so, by (i), $\operatorname{dim} X_{g}=\operatorname{dim} Z(G)$. Since $g X_{g}=(g Z(G)) \cap X$, we have, for every $g \in \operatorname{Tor}(G)$,

$$
\operatorname{dim} g Z(G) \cap X=\operatorname{dim} Z(G) .
$$

Let

$$
X_{1}=\{g \in G: \operatorname{dim}(g Z(G) \cap X)=\operatorname{dim} Z(G)\} .
$$

It follows from the above that $\operatorname{Tor}(G) \subseteq X_{1}$, and therefore $\operatorname{Tor}(G / Z(G)) \subseteq$ $X_{1} / Z(G)$. (Indeed, if $h Z(G) \in \operatorname{Tor}(G / Z(G))$ then for some $n \in \mathbb{N}, h^{n} \in$ $Z(G)$, and therefore for some $k \geq n, h^{k} \in Z(G)^{0}$. Because $Z(G)^{0}$ is divisible there exists $h_{1} \in Z(G)$, with $h_{1}^{k}=h^{k}$. Because $h_{1}$ is central, 
$\left(h h_{1}^{-1}\right)^{k}=e$, hence $h h_{1}^{-1} \in X_{1}$ and therefore $\left.h Z(G) \in X_{1} / Z(G)\right)$. By (ii), $\operatorname{dim}\left(X_{1} / Z(G)\right)=\operatorname{dim}(G / Z(G))$.

To finish the proof, consider the set $Y=\left(X_{1} Z(G)\right) \cap X$. The cosets of $Z(G)$ partition $Y$ into equivalence classes, each of $\operatorname{dimension} \operatorname{dim} Z(G)$ (by definition of $X_{1}$ ). Since every $Z(G)$-coset of an element in $X_{1}$ intersects $X$ nontrivially, we have

$$
\operatorname{dim}(Y / Z(G))=\operatorname{dim}\left(X_{1} / Z(G)\right)=\operatorname{dim}(G / Z(G)) .
$$

Summarizing, we have

$$
\operatorname{dim} Y \geq \operatorname{dim} Z(G)+\operatorname{dim}(G / Z(G))=\operatorname{dim} G,
$$

and hence (since $Y \subseteq X) \operatorname{dim} X=\operatorname{dim} G$.

\section{References}

[1] A.Berarducci, Zero-groups and maximal tori in Logic Colloquium'04, A. Andretta et al. (eds.),ASL Lecture Notes in Logic, Vol. 29 (2006) 33-45

[2] M.Edmundo, A remark on divisibility of definable groups, Math. Logic Quart.51 (2005) 639-641.

[3] M.Edmundo and M.Otero, Definably compact abelian groups, J. Math. Logic 4 (2004) 163-180.

[4] E.Hrushovski, Y.Peterzil and A.Pillay, Groups, measures and NIP, J. Am. Math.Soc., 34pp. to appear.

[5] Y.Peterzil, A.Pillay and S.Starchenko, Definably simple groups in ominimal structures, Trans. Am. Math. Soc. 352 (2000) 4397-4419.

[6] Y.Peterzil, A.Pillay and S.Starchenko, Simple algebraic and semialgebraic groups over real closed fields, Trans.AMS 352 (2000) 4421-4450.

[7] Y.Peterzil, A.Pillay and S.Starchenko, Linear groups definable in ominimal structures, J. Algebra 247 (2002) 1-23.

[8] Y.Peterzil and S.Starchenko, Definable homomorphisms of abelian groups in o-minimal structures, Ann. Pure Appl. Logic 101 (2000) 1-27. 